\title{
Development of a method to determine the cost of breast cancer treatment with chemotherapy at Groote Schuur Hospital, Cape Town, South Africa
}

\author{
N T Guzha, ${ }^{1}$ BPharm, MPharm; T Thebe, ${ }^{2}$ MB ChB, FC Rad Onc (SA), MMed (Radiation Therapy); N Butler, ${ }^{1}$ BPharm, MPharm, PhD; \\ P N Valodia, ${ }^{1}$ BPharm, MPharm, PhD \\ ${ }^{1}$ School of Pharmacy, Faculty of Natural Sciences, University of the Western Cape, Cape Town, South Africa \\ ${ }^{2}$ Department of Radiation Oncology, Groote Schuur Hospital and Faculty of Health Sciences, University of Cape Town, South Africa
}

Corresponding author: P N Valodia (praneetvalodia@gmail.com)

Background. There has been no comprehensive study determining the financial burden of breast cancer in the South African (SA) public sector. Objectives. To develop a method to determine the cost of breast cancer treatment with chemotherapy per episode of care and to quantify the associated costs relating to chemotherapy at Groote Schuur Hospital (GSH), a government hospital in SA. These costs included costs associated with the management of adverse events arising from chemotherapy.

Methods. Retrospective patient-level data were collected for 200 patients from electronic databases and patient folders between 2013 and 2015. Direct medical costs were determined from the health funder's perspective. The information collected was categorised into the following cost components: chemotherapy medicines, support medicines, administration of chemotherapy, laboratory tests, radiology scans and imaging, doctor consultations and adverse events. Time-and-motion studies were conducted on a set of new patients and the data obtained were used for the study sample of 200 patients. All the above costs were used to determine the cost of chemotherapy per episode of care. The episode of care was defined as the care provided from 2 months prior to the date of commencing chemotherapy (prechemotherapy phase), during chemotherapy (treatment phase) and until 6 months after the date when the last cycle of chemotherapy was administered (follow-up phase).

Results. A method was developed to determine the episode-of-care costs for breast cancer at GSH. The total direct medical cost for treatment of breast cancer at GSH for 200 patients was ZAR3 154 877, and the average episode-of-care cost per patient was ZAR15 774. The average cost of management of adverse events arising from the various treatment modalities was ZAR13 133 per patient. It was found that the cost of treating a patient with adverse events was 1.8 times higher than the cost of treating a patient without adverse events. Of the patients, $86.5 \%$ managed to complete their prescribed chemotherapy treatment cycles, and the average cost of treatment of these patients was 1.3 times more than the average cost for patients who could not complete their treatment, based on the number of treatment cycles received.

Conclusion. A comprehensive method to determine the costs associated with breast cancer management per episode of care was developed, and costs were quantified at GSH according to the treatment protocol used at the hospital.

S Afr Med J 2020;110(4):296-301. https://doi.org/10.7196/SAMJ.2020.v110i4.14204

Breast cancer is one of the major healthcare challenges in South Africa (SA). Data published by the National Cancer Registry ${ }^{[1]}$ in 2014 showed that breast cancer represented $22 \%$ of all cancers affecting women in SA, making it the most prevalent cancer affecting women in this country. Understanding the total cost of care for breast cancer is important from a health funder perspective, i.e. policymakers and hospital administration, in budget allocation and in decision-making ${ }^{[2]}$ in a resource-constrained environment.

\section{Objectives}

To develop a method to determine the cost of breast cancer treatment with chemotherapy per episode of care and to quantify the associated costs relating to chemotherapy at Groote Schuur Hospital (GSH), a tertiary government hospital in Western Cape Province, SA. These costs included costs associated with the management of adverse events arising from chemotherapy.

\section{Methods}

Study design

A retrospective cohort analysis was performed to determine the cost of an episode of care for treatment of breast cancer with chemotherapy. Cost estimates for an episode of care were obtained for each stage of breast cancer. The episode of care was defined as the care provided from 2 months prior to the date of commencing chemotherapy (pre-chemotherapy phase), during chemotherapy (treatment phase) and until 6 months after the date when the last cycle of chemotherapy was administered (follow-up phase). The episode of care was on average a period of $10-12$ months per patient.

\section{Participants}

A total of 1024 patients were extracted from the electronic database. Two hundred patients were randomly selected using a random number generator. Patients were included in the study based on the following criteria: (i) a diagnosis of breast cancer; (ii) registration date at the oncology department from 1 April 2013 to 31 March 2015; (iii) evidence of receipt of chemotherapy with two or more health encounters ${ }^{[3]}$ related to breast cancer, e.g. doctor consultation in oncology ward and a mammogram; and (iv) age $\geq 18$ years. Patients were excluded from the study based on the following criteria: (i) male; (ii) initiated on hormone therapy but not chemotherapy; (iii) enrolled in a clinical trial; and (iv) a primary diagnosis of cancer that was not breast cancer. 


\section{Setting}

The study site was GSH, a tertiary government hospital in the Western Cape.

\section{Development of method}

Patient information obtained from the folders was recorded on data collection forms. In instances where patient folders were missing during the data collection period, another patient folder was randomly selected to replace the missing folder. The associated electronic patient profile was also extracted. Each patient in the study had electronic and folder data collected.

The electronic database provided the following information: (i) patient demographics, i.e. name, surname and hospital number; (ii) medicine profile, which included the name, date of issue, quantity issued and cost of each chemotherapy medicine; (iii) support medicines, which included the name, dose, cost and date of issue of each medicine; (iv) laboratory tests, which included the name of the test, number of tests performed and date when the test was performed; $(v)$ scans and imaging data, which included the name, number and date of scans and imaging performed; ( $v i)$ consultations, which included scheduled and emergency visits, date of consultation and department attended by the patient; and (vii) inpatient hospitalisation data, which included the date of admission, date of discharge, duration of stay, ward type and reason for admission.

The patient folders provided data to supplement the information obtained from the electronic database. Folder information was used to verify the electronic data. Additional information received from the folders included age at diagnosis, date of registration in the Radiation Oncology Department, stage of disease, weight, height and body surface area. Detailed clinical information collected included the histological type of disease, comorbidity, treatment intent, hormone receptor status, doctors' notes on the consultations, and adverse events data, which included the date when the event occurred, associated emergency consultation, inpatient hospitalisation and reason for admission.

\section{Costing method}

Only direct medical costs and resource use relating to chemotherapy in the management of breast cancer at GSH were used in this study. In addition, the costs of management of adverse events were determined. The various cost components were aggregated for calculation of the total cost of an episode of care.

The sources of costs used in this study were the Medical Informatics electronic database, the JAC Medicines Management system, the Uniform Patient Fee Schedule (UPFS), ${ }^{[4]}$ and time-and-motion studies. The facility and professional fees for administration of chemotherapy, scheduled outpatient consultation, emergency outpatient consultation, inpatient hospitalisation, scans and imaging, and laboratory tests were obtained from the UPFS. The UPFS was also the source of the medicine dispensing fee and the cost of drawing blood for laboratory tests. According to the UPFS, the single professional fee consisted of the cost of the doctors, nurses and other healthcare professionals involved in the care of the patient. The facility fee was a fee charged for the environment and equipment used for providing the service to the patient.

The cost of adverse events applied only to patients who had a record of serious adverse event(s) during the episode of care. In this study, the definition of a serious adverse event was any unwanted or unexpected reaction requiring hospitalisation or emergency consultation resulting from treatment with chemotherapy for breast cancer. The cost components that contributed to the cost of treating adverse events were support medicines, laboratory tests, emergency consultations and inpatient hospitalisation costs charged per day for the full length of stay. The cost of breast cancer treatment and associated adverse events was broadly categorised based on the stage at diagnosis, i.e. I, II, III or IV according to tumour-node-metastasis (TNM) staging. ${ }^{[5]}$

Table 1 presents the process that was followed when calculating the cost of components.

\section{Time-and-motion studies}

The costs of compounding of chemotherapy, administration of chemotherapy and outpatient doctor consultations were also calculated using data obtained from time-and-motion studies. A data collection form was used to record the times taken to perform these tasks. The time for each activity was multiplied by the per-minute salary. ${ }^{[6]}$ The salaries of the pharmacists, doctors and nurses were derived from information published by the Department of Public

\begin{tabular}{ll} 
Table 1. Calculation of cost components \\
\hline Cost component & Cost calculation \\
\hline Chemotherapy medicines & Cost of medicines per cycle, which were added to provide the total cost for all treatment cycles \\
& + \\
& Dispensing fee unit cost multiplied by the number of treatment cycles. The dispensing fee was added only \\
once for each patient for each day a prescription was handled at the pharmacy & Cost of all support medicines \\
& + \\
Support medicines & Dispensing fee, where applicable \\
& Professional fee per treatment cycle multiplied by the number of treatment cycles \\
& + \\
Chemotherapy administration & Facility fee per treatment cycle multiplied by the number of treatment cycles \\
& Cost of doctor consultation multiplied by the number of consultations during the episode of care \\
& + \\
Consultations & Facility fee per consultation multiplied by the number of consultations during the episode of care \\
& Sum of unit costs for each test conducted \\
& + \\
Laboratory tests & Fee for drawing of blood, calculated for each day laboratory tests were done (usually at every cycle of \\
& chemotherapy) \\
& Sum of unit costs for each procedure conducted \\
& + \\
& Sum of facility fee for each procedure conducted
\end{tabular}


Service and Administration of SA. ${ }^{[7]}$ For all these studies, the observer used a digital stopwatch for accurate capture of the time taken to complete a task.

\section{Statistical analysis}

Descriptive statistics were used for all variables. Both means and medians were used to present cost of treatment owing to some outliers that made the means differ from the median. Wilks' lambda test was conducted to test whether there were significant differences in costs between the stages of breast cancer. A $p$-value $<0.05$ was considered to be statistically significant.

\section{Ethical considerations}

Ethics approval for the study was obtained from the Research Ethics Committee of the University of the Western Cape (ref. no. 14/9/48) and the Human Research Ethics Committee of the University of Cape Town (HREC ref. no. 824/2014). Patient confidentiality was maintained at all times.

\section{Results}

The mean (standard deviation) age of the breast cancer patients was 50 (11.6) years (range 23 - 77 years). The incidences of the various stages of breast cancer are reported in Table 2. Of all the stages of breast cancer, the highest incidence (49\%) was reported for stage II at the point of registration, as reflected in Table 2. The lowest incidence (7\%) was recorded for stage I breast cancer. We found that at least $44 \%$ of patients were diagnosed with late-stage cancer, i.e. stages III and IV combined. Of the patients, 55\% presented with a pre-existing comorbidity at diagnosis, and of these $27 \%$ had two or more comorbid conditions. The most frequent comorbidities were

\begin{tabular}{|c|c|}
\hline Patient characteristics & Patients $(N=200), n(\%)$ \\
\hline \multicolumn{2}{|l|}{ Age at diagnosis (years) } \\
\hline $18-35$ & $22(11)$ \\
\hline $36-45$ & $46(23)$ \\
\hline $46-65$ & $113(56.5)$ \\
\hline$>65$ & $19(9.5)$ \\
\hline \multicolumn{2}{|l|}{ Stage at diagnosis } \\
\hline I & $14(7)$ \\
\hline II & $98(49)$ \\
\hline III & $65(32.5)$ \\
\hline IV & $23(11.5)$ \\
\hline \multicolumn{2}{|l|}{ Histology } \\
\hline Ductal DCIS, IDC & $169(84.5)$ \\
\hline Lobular LCIS, ILC & $13(6.5)$ \\
\hline Paget's disease & $1(0.5)$ \\
\hline Mucinous & $1(0.5)$ \\
\hline Adenoma NOS & $1(0.5)$ \\
\hline Not mentioned & $15(7.5)$ \\
\hline \multicolumn{2}{|l|}{ Treatment modality } \\
\hline Adjuvant & $123(61.5)$ \\
\hline Neo-adjuvant & $59(29.5)$ \\
\hline Palliative & $18(9)$ \\
\hline \multicolumn{2}{|l|}{ Comorbidity } \\
\hline 0 & $91(45.5)$ \\
\hline 1 & $55(27.5)$ \\
\hline$\geq 2$ & $54(27)$ \\
\hline
\end{tabular}

heart diseases (35.5\%), endocrine diseases (12.5\%), arthritis and pulmonary diseases ( $8 \%$ each) and HIV (5\%). Other less common comorbidities were depression, eczema and epilepsy.

\section{Cost of chemotherapy}

The three aspects of chemotherapy costs that were studied were the cost of chemotherapy medicines, the dispensing fee and the cost of administration of chemotherapy. The cost of intravenous chemotherapy medicines was ZAR761 152 for the 200 patients. The cost of chemotherapy and the dispensing fee amounted to ZAR801 311 for the full sample. The cost of administering chemotherapy to the 200 patients was ZAR262 749. This cost consisted of a professional fee and a facility fee. The dispensing fee of ZAR36 per patient per prescription was $5 \%$ of the cost of chemotherapy medicines. The cost of chemotherapy medicines was 2.9 times more than the cost of administering the chemotherapy. This information provided an indication of the relationship between the various costs. Of the patients, $86.5 \%$ managed to complete their prescribed chemotherapy treatment cycles, and the average cost of treatment of these patients was 1.3 times more than the average cost for patients who could not complete their treatment, based on the number of treatment cycles received.

\section{Episode-of-care costs}

The episode-of-care costs and the contribution of the various cost components are summarised in Table 3 .

\section{Cost of management of breast cancer}

The average costs of managing patients diagnosed with breast cancer at GSH are reported in Table 4. The average cost was calculated based on each patient's duration of treatment. The average costs for patients who completed and did not complete treatment were categorised separately. The average cost of treating a patient who adhered to the prescribed chemotherapy protocol for the full duration of treatment was ZAR16 259. The average cost for the small proportion (13.5\%) of the study sample who did not complete the prescribed treatment was ZAR12 664 per patient. The combined average cost for the 200 patients in the study was ZAR15 774. The mean and median costs per patient, based on whether the protocol was completed or not, are shown in Table 4.

Of the sample of 200 patients, 16 had adverse events arising from chemotherapy that led to additional health resource utilisation. The most commonly occurring adverse event was neutropenia. The cost of treating a patient with neutropenia was ZAR36 465 . For the sample of 200 patients, the total cost of managing adverse events was ZAR210 128; this cost was attributed to 16 patients experiencing a total of 27 adverse events, and included the cost of hospitalisation, intravenous antibiotics and anti-emetics. The average cost of management of adverse events arising from the various treatment modalities was ZAR13 133 per patient. The maximum length of hospital stay for the treatment of adverse events was 15 days, costing ZAR42 672 for the hospital stay alone.

\section{Time-and-motion results}

It took an average of $9.8,6.7$ and 5 minutes to compound 5-fluorouracil, epirubicin and cyclophosphamide (FEC), epirubicin and cyclophosphamide (EC) and paclitaxel (P), respectively. The results were used to calculate the cost of compounding of chemotherapy by the pharmacist. The cost of compounding for the episode of care for 200 patients was ZAR44 671. The cost of chemotherapy compounding increased the total cost of medicines by $5.3 \%$.

It took an average of 16.9 minutes of nurses' time to administer chemotherapy. However, the time varied according to the regimen 
Table 3. Summary of episode-of-care costs (ZAR) for each component for the treatment of breast cancer at Groote Schuur Hospital

\begin{tabular}{|c|c|c|c|c|c|}
\hline \multirow[b]{2}{*}{ Cost component } & \multicolumn{5}{|c|}{ Costs for 10 - 12-month period ( $N=200$ patients) } \\
\hline & Cost $(\%)$ & Mean cost per patient & SD & Median & Range \\
\hline Chemotherapy and dispensing fee & $801311(25.4)$ & 4006 & 1635 & 3880 & $544-10653$ \\
\hline Chemotherapy administration & $262749(8.3)$ & 1313 & 335 & 1404 & $234-3276$ \\
\hline Support medicines & $235425(7.5)$ & 1177 & 738 & 1089 & $116-7385$ \\
\hline Consultations & $549562(17.4)$ & 2747 & 736 & 2691 & $897-4784$ \\
\hline Laboratory tests (routine) & $445247(14.1)$ & 2237 & 1166 & 1997 & $447-6840$ \\
\hline Scans and imaging (routine) & $860583(27.3)$ & 4302 & 1674 & 4233 & $527-10064$ \\
\hline Total cost & $3154877(100)$ & - & - & - & - \\
\hline
\end{tabular}

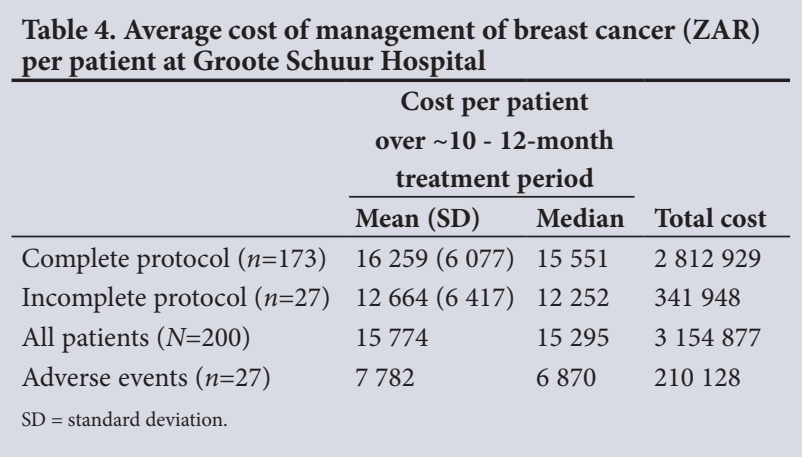

of chemotherapy received. It took an average of 19.5 minutes to administer FEC, 13.8 minutes for EC and 17.3 minutes for $\mathrm{P}$ (Table 5). The single-dose regimen $\mathrm{P}$ took 3.5 minutes more than the EC regimen because the infusion rate of the $\mathrm{P}$ regimen was slower than the EC regimen. These results do not reflect infusion time alone but the actual contact time of the nurse with the patient with regard to chemotherapy administration.

The average doctors' time for the first and follow-up consultations was 20.5 and 12.1 minutes, respectively. The cost per cycle for the former was 1.7 times more that the latter, i.e. ZAR114 v. ZAR68.

\section{Cost of breast cancer based on clinical stage}

The mean cost per patient for the various stages is presented in Table 6. Analysis of stage at diagnosis revealed that breast cancer patients who presented at stages III and IV had the highest cost of treatment based on the mean cost per patient per stage. These costs included all patients, whether the prescribed protocol of chemotherapy was completed or not.

For the cohort of 200 patients, the total cost of treating early-stage cancer was ZAR1 670429 and that of treating late-stage cancer was ZAR1 484 447. The higher total cost of early-stage cancer treatment was due to the greater number of patients with early breast cancer $(n=112)$ v. late breast cancer $(n=88)$. In this study, early-stage breast cancer was categorised as stages I and II disease, while late-stage breast cancer was categorised as stages III and IV disease. Wilks' lambda test found significant differences in cost between stages I and III, stages I and IV and stages II and III $(p<0.001)$. However, no statistically significant difference was observed between stages I and II and stages III and IV ( $p>0.05)$.

Cost of breast cancer treatment based on age at diagnosis When patients were grouped according to age, without consideration of the type of chemotherapy received or the severity of their cancer, the highest number of cases of breast cancer $(n=113)$ was in the 46 - 65 years age group and the lowest number $(n=19)$ in the oldest age group, $>65$ years (Table 7 ). The cost of treatment per age group is shown in Table 7. The reported differences of the average cost per patient in each age group were found not to be statistically significant following Wilks' lambda test $(p=0.15)$.

\section{Discussion}

This study focused on the development of a method to determine the cost of breast cancer treatment with chemotherapy at GSH. It addressed a common limitation of published studies, where the development of costing methods is not presented in sufficient detail to make methods transparent and reproducible. ${ }^{[8]}$

The strength of this cost study lies in the determination of actual costs of treatment at patient level for an episode of care. Utilisation data in the routine setting allowed costing of chemotherapy without making assumptions of usage. This was crucial in contrast to other studies that make assumptions of cost based purely on the protocols being followed.

No direct comparisons between different countries was made in this evaluation owing to differences in population, cancer incidence, socioeconomic status and health funding systems. An important objective of this study was to develop a method for GSH to determine the cost of breast cancer for an episode of care. Studies have been done in other countries such as Canada ${ }^{[9]}$ and Morocco, ${ }^{[10]}$ but are not comparable owing to different costs and methods used.

The major cost driver in this study was scans and imaging, followed by a combination of chemotherapy and dispensing fee costs. Procedure and facility fees per patient for scans and imaging were higher when compared with other component costs. The reason for this could be the use of high-technology machines that required a specialist radiographer and experienced personnel to interpret the results. The cost of the isotopes was included in this analysis. The cost of maintenance of these machines is a challenge in developing countries. ${ }^{[11]}$

The costs of chemotherapy medicines were the second highest of all the cost components. A huge variation in cost of chemotherapy within the same patient's episode of care for the same medicines and the same dosage was observed. The variation in chemotherapy prices was mainly accounted for by the variation in the batch size compounded on the particular day.

The costs of breast cancer treatment were dependent on a variety of factors, i.e. stage at diagnosis, adverse events and complete or incomplete treatment. We found that there was no statistical difference between the various age groups when episode-of-care costs were compared. In our study, the average age at diagnosis was 50 years and at least $50 \%$ of patients were $>50$ years old. The age group 46 - 65 years accrued the highest cost of treatment owing to the high number of patients in this age group (Table 7). Lan et $a l .{ }^{[12]}$ similarly reported that there were no significant differences in median total cost related to patient age $(p=0.329)$. 


\begin{tabular}{lll} 
Table 5. Time-and-motion study results & & \\
\hline Task & Mean time per cycle (min) & Mean cost per cycle (ZAR) \\
\hline Compounding of chemotherapy & Pharmacists' time & 44 \\
FEC & 9.8 & 31 \\
EC & 6.7 & 23 \\
P & 5.0 & \\
Consultation & Doctors' time & 114 \\
First visit & 20.5 & 68 \\
Follow-up & 12.1 & 39 \\
Administration of chemotherapy & Nurses' time & 27 \\
FEC & 19.5 & 34 \\
EC & 13.8 & \\
P & 17.3 & 7 \\
Administration of chemotherapy & Doctors' time & \\
Updating patient folder/responding to queries & 1.3 & \\
F = 5-fluorouracil; E = epirubicin; C = cyclophosphamide; $\mathrm{P}=$ paclitaxel. & &
\end{tabular}

Table 6. Cost of treatment (ZAR) per stage of breast cancer for all patients

\begin{tabular}{llll}
\hline Stage at diagnosis & Total cost per stage & Mean cost per patient per stage & Range \\
\hline I & $191050(n=14)$ & 13646 & $5611-24419$ \\
II & $1479379(n=98)$ & 15095 & $3480-37705$ \\
III & $1096615(n=65)$ & 16871 & $3922-38627$ \\
IV & $387832(n=23)$ & 16862 & $5867-36569$
\end{tabular}

Table 7. Cost of treatment (ZAR) per age group

\begin{tabular}{llll}
\hline Age at diagnosis (years) & Cost per age group & Cost per patient per age group & Range \\
\hline $18-35(n=22)$ & 358815 & 16309 & $5635-33232$ \\
$36-45(n=46)$ & 737405 & 16030 & $4047-40932$ \\
$46-65(n=113)$ & 1782553 & 15774 & $2821-39076$ \\
$>65(n=19)$ & 280463 & 14761 & $6345-22294$
\end{tabular}

It was found in the present study that the stage of cancer at diagnosis was probably the most crucial parameter in defining the cost of treatment. The mean cost of treatment for stage I was significantly lower than the cost for more advanced stages. An early diagnosis of cancer (particularly stage I) therefore results in a lower cost of treatment compared with late-stage breast cancer (stages III and IV). Another study ${ }^{[12]}$ has also reported that the progress of disease was the main factor influencing the cost of treatment, because medical intervention was decided on according to the extent of spread and stage of disease. The cost of late-stage treatment was increased by the more likely occurrence of adverse events, treatment complications and palliative care. ${ }^{[12]}$ As adverse effects contribute to extensive resource use in hospital, it is important that these costs are considered. ${ }^{[13]}$ Our study, like that of Lan et al., ${ }^{[12]}$ showed that costs of treatment are reduced when breast cancer is diagnosed at an early stage and that age at diagnosis did not significantly influence the cost of treatment.

To improve the accuracy of the costing, time-and-motion studies were conducted. The cost of compounding chemotherapy increased the total cost of chemotherapy by $5.3 \%$. It appears that the UPFS did not account for the costs associated with the compounding of chemotherapy. Our study therefore considered this additional cost. This cost component should be accounted for, as it is an important cost of treatment. ${ }^{[6]}$

With regard to the time-and-motion studies, the time recordings were conducted by the researcher and not self-reported. This approach reduced the risk of bias and systematic misreporting of time spent. The recording of actual times provided an opportunity to measure actual resource use as opposed to calculating costs from the protocol-based method. ${ }^{[14]}$

\section{Study limitations}

While the sample size of 200 patients was sufficient to develop a costing method for breast cancer at GSH, the sample size may not be large enough to conduct detailed statistical analyses owing to the smaller sample sizes in the sub-population groups.

\section{Conclusions}

A method of costing breast cancer treatment at GSH was successfully developed. The costs associated with breast cancer management per episode of care were quantified at GSH according to the treatment protocols used at the hospital. The average cost per patient for an episode of care was ZAR15 774, and the average cost of managing an adverse event was ZAR7 782. These results may not be generalisable to other public hospitals. Understanding the total cost of care for breast cancer is important in SA owing to the high prevalence of breast cancer relative to other cancers and budgetary constraints. With the advent of National Health Insurance in SA, understanding the total cost of care will become even more important to improve budget allocation. The mean cost of treatment for stage I breast cancer was significantly lower than that for the more advanced stages. An early diagnosis of cancer (particularly stage I) therefore results 
in a lower cost of treatment compared with late-stage breast cancer (stages III and IV).

Declaration. The research for this study was done in partial fulfilment of the requirements for NTG's MPharm degree at the University of the Western Cape.

Acknowledgements. The authors thank Dr Leon van Wijk, Department of Radiation Oncology, GSH, for clinical support and Ms Wendy Bryant and Ms Salwa Kriel, Medical Informatics Department, GSH, for the extraction of data from the hospital database.

Author contributions. The article was written by PNV and NTG and reviewed by NB and TT.

Funding. None.

Conflicts of interest. None.

1. National Cancer Registry, South Africa. 2014. http://www.nicd.ac.za/wp-content/uploads/2017/03/2014NCR-tables-1.pdf (accessed 8 January 2019).

2. Pompen M, Gok M, Novák A, et al. Direct costs associated with the disease management of patients with unresectable advanced non-small-cell lung cancer in The Netherlands. Lung Cancer 2009;64(1):110 116. https://doi.org/10.1016/j.lungcan.2008.07.009

3. Vera-Llonch M, Weycker D, Glass A, et al. Healthcare costs in women with metastatic breast cancer receiving chemotherapy as their principal treatment modality. BMC Cancer 2011;11:1-7. http://www. biomedcentral.com/1472-6963/11/305 (accessed 8 January 2019).
4. Western Cape Government, South Africa. Amendments to the Uniform Patient Fee Schedule. Western Cape Provincial Gazette No. 7245, 17 March 2014. https://archive.opengazettes.org.za/archive/ZAWC/2014/provincial-gazette-ZA-WC-no-7245-dated-2014-03-27.pdf (accessed 8 January 2019).

5. Davari M, Yazdanpanah F, Aslani A, Hosseini M, Nazari AR, Mokarian F. The direct medical costs of bavari M, Yazdanpanah F, Aslani A, Hosseini M, Nazari AR, Mokarian F. The direct medical costs of
breast cancer in Iran: Analyzing the patient's level data from a cancer specific hospital in Isfahan. Int Jreast cancer in Iran: Analyz

6. Oglesby A, Sherif B, Odom D, Leahy M, Qian Y. Time and costs associated with preparing and administering zoledronic acid in patients with breast or prostate cancer and metastatic bone disease. Community Oncol 2009;6(11):494-502.

7. Department of Public Service and Administration, South Africa. Salary scales, with translation keys, for employees on salary levels 1 to 12 and covered by Occupation Specific Dispensations (OSDs): Costof-living adjustment with effect from 1 April 2014. 2014. https://www.westerncape.gov.za/text/2014/ May/salary-scales-2014.pdf (accessed 8 January 2019).

8. Cipriano LE, Romanus D, Earle CC, et al. Lung cancer treatment costs, including patient responsibility, by disease stage and treatment modality, 1992 to 2003. Value Health 2011;14(1):41-52. https://doi. org/10.1016/j.jval.2010.10.006

9. De Oliveira C, Bremner KE, Pataky R, et al. Trends in use and cost of initial cancer treatment in Ontario: A population-based descriptive study. CMAJ 2013;1(4):E151-E158. https://doi.org/10.9778/ cmajo.2013004110.9778/cmajo.20130041

10. Boutayeb S, Boutayeb A, Ahbeddou N, et al. Estimation of the cost of treatment by chemotherapy or early breast cancer in Morocco. Cost Eff Resour Alloc 2010;8(16):1-6. https://doi. org/10.1186\%2F1478-7547-8-16

11. Shah S, Bellows BA, Adedipe AA, et al. Perceived barriers in the use of ultrasound in developing countries. Crit Ultrasound J 2015;7(1):1-5. https://doi.org/10.1186/s13089-015-0028-2

12. Lan NH, Laohasiriwong W, Stewart JF, Tung ND, Coyte PC. Cost of treatment for breast cancer in central Vietnam. Glob Health Action 2013;6(1):1-10. https://doi.org/10.3402/gha.v6i0.18872

13. Pettersson K, Carlsson G, Holmberg C, Sporrong SK. Cost identification of Nordic FLIRI, Nordic FLOX, XELIRI and XELOX in first-line treatment of advanced colorectal cancer in Sweden - a clinical practice model approach. Acta Oncol 2012;51(7):840-848. https://doi.org/10.3109/028418 6X.2012.713507

14. Haywood P, de Raad J, van Gool K, et al. Chemotherapy administration: Modelling the costs of alternative protocols. Pharmacoeconomics 2012;30(12):1173-1186.

Accepted 17 September 2019. 